\title{
A methodology for momentum flux measurements in two-phase blast flows
}

\author{
R. G. Ames ${ }^{1} \&$ M. J. Murphy ${ }^{2}$ \\ ${ }^{I}$ Naval Surface Warfare Center, Dahlgren Division, USA \\ ${ }^{2}$ Lawrence Livermore National Laboratory, USA
}

\begin{abstract}
Modern diagnostics for air blast waves have been developed to the point that they are sufficiently accurate and robust to capture most blast phenomena of interest (most often pressure). Two-phase blast flows also transport momentum and energy in a non-gas phase and, as such, the properties of this second phase must be accounted for in estimating flowfield parameters. Standard air blast diagnostics are not sufficient to capture these effects and the diagnostics that do exist for multiphase flows normally rely on steady conditions or sampling periods of at least a few milliseconds. The extreme transients associated with multiphase blast flows (microsecond-scale) preclude the use of such techniques. For this reason, novel approaches are required in order to capture the total momentum and energy flux in two-phase blast flows. This paper outlines a novel suite of diagnostics and an analysis technique that allows for momentum flux measurements in two-phase blast flows.
\end{abstract}

Keywords: multiphase blast, two-phase blast, diagnostics, momentum flux, impulse.

\section{Introduction}

The problem of separate momentum flux measurements in two-phase blast flows is difficult because of the difference in length scales over which the two phases interact with measurement devices. When trying to measure a reflected pressure using a standard pressure gauge, the gas-phase loads are relatively constant over the sensor surface area. As such, the average load is very close to the local loads across the sensing surface. For a solid-phase particle blast, the local gradients are very large due to particle impacts against the sensing surface. The average load across the sensing surface is much lower, however, because the particles 
appear at discrete locations with spacing that is too large to be considered continuum. As such, the average load across the gauge sensing surface might be well within the calibrated range of a standard pressure gauge but the local loads are much higher and are likely to produce damage to the sensing surface. For this reason, novel diagnostic techniques for two-phase blast flows have been developed and documented in a previous publication [1].

While it is relatively straightforward to collect integrated measurements of multiple-phase blast effects (e.g. impulse measurements using the momentum trap technique) it is more difficult to extract the time-history of the loading that produces that impulse. As explained above, standard pressure gauges, which are sufficient to obtain detailed time-histories for gas-phase loads, are not suitable for flowfields that contain a significant amount of solid particulate. In order to facilitate this type of measurement it is often necessary to measure parameters such as the number density flux of solid-phase particles. This type of measurement, combined with measurements of the time-varying speed of the particles, will give a measure of the momentum and energy flux in the solid phase. If the speed of the particles cannot be measured (as is usually the case) then their behavior can be estimated using a suitable model with parameters derived from measurable quantities. In either case, the combination of timevarying and integrated measurements serves to increase the accuracy of the technique. This paper describes one approach to the momentum flux measurement problem using a combination of number density flux measurements and impulse measurements.

\section{Measurement techniques}

The methodology described here uses two main measurements: one for the number density flux of particles and one for the impulse delivered to a rigid target. The number density flux is measured using a particle streak recorder that uses a rotating drum placed in a multiphase blast field to produce a time history of the particulate passing through a thin aperture. The impulse measurements are performed using the momentum trap technique.

\subsection{The momentum trap technique}

The momentum trap technique was first described by Hopkinson in his classic paper describing the pressure bar technique for measuring blast and impact loads [2]. More recently, Held [3, 4] applied a similar technique to the problem of measuring the impulse generated by air blast waves against cylindrical targets. The Held technique uses a block of known mass and geometry suspended above a level surface at some distance $d$ from an explosive charge. When the charge is detonated and the blast wave produces loads on the block, it is thrown from its initial location. So long as the time scales associated with the blast loads are much less than the time scales associated with the motion of the block it can be assumed that the load is impulsive. As such, the block of mass $m_{b}$ will be instantly accelerated to a speed $v$ in the direction of the blast wave motion. 


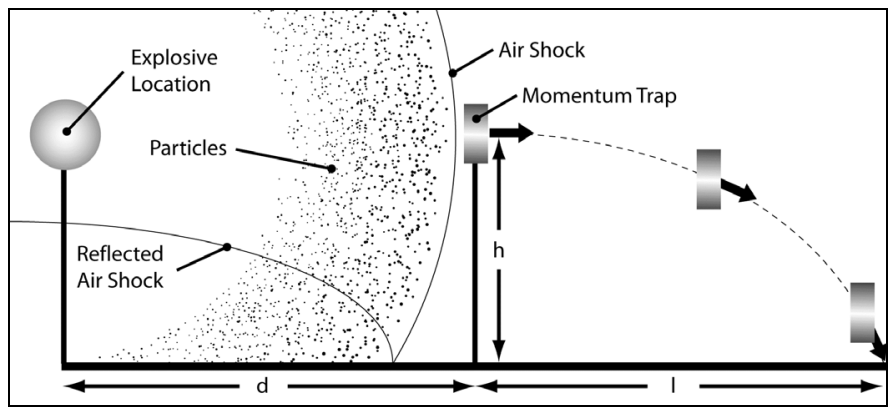

Figure 1: Diagram showing the momentum trap measurement technique.

So long as the speed of the block is sufficiently low it will travel ballistically to a landing distance $l$ from its initial location. Given the known initial center of gravity height $h$ it can be shown that the change in momentum of the block (which is equal to the impulse delivered to it) is given by

$$
I=\frac{m_{b} l}{\sqrt{2 h / g}}
$$

where $g$ is the acceleration due to gravity. It is usually the case that the impulse is normalized to the frontal surface area of the block to give a measure of the impulse per unit area exposed to the blast. This area, $A$, and impulse per unit area $I_{A}$ are then related by the expression

$$
I_{A}=\frac{\phi l}{\sqrt{2 h / g}}
$$

where $\phi$ is the frontal area density of the block, $m_{b} / A$. The measurement problem, then, reduces to the problem of measuring the throw distance $l$. This is usually achieved by placing the momentum trap within a level sand pit: when the block is thrown from its initial location it impacts on the sand and leaves a mark to indicate the throw distance.

\subsection{The particle streak recorder}

The particle streak recorder is a device that measures the flux of particles in a two-phase blast flow. It does so by employing a spinning drum within a protective shroud. The shroud includes a thin aperture that is aligned toward the oncoming blast wave. As the two-phase blast wave passes the front of the shroud, the aperture allows a small amount of particulate to pass onto the rotating drum. The rotating drum is designed such that the surface will allow the particulate to leave an impression as the disc is spinning. As a result, the drum produces a time history of the particulate that passes through the aperture via the record of particle impacts across its surface. 

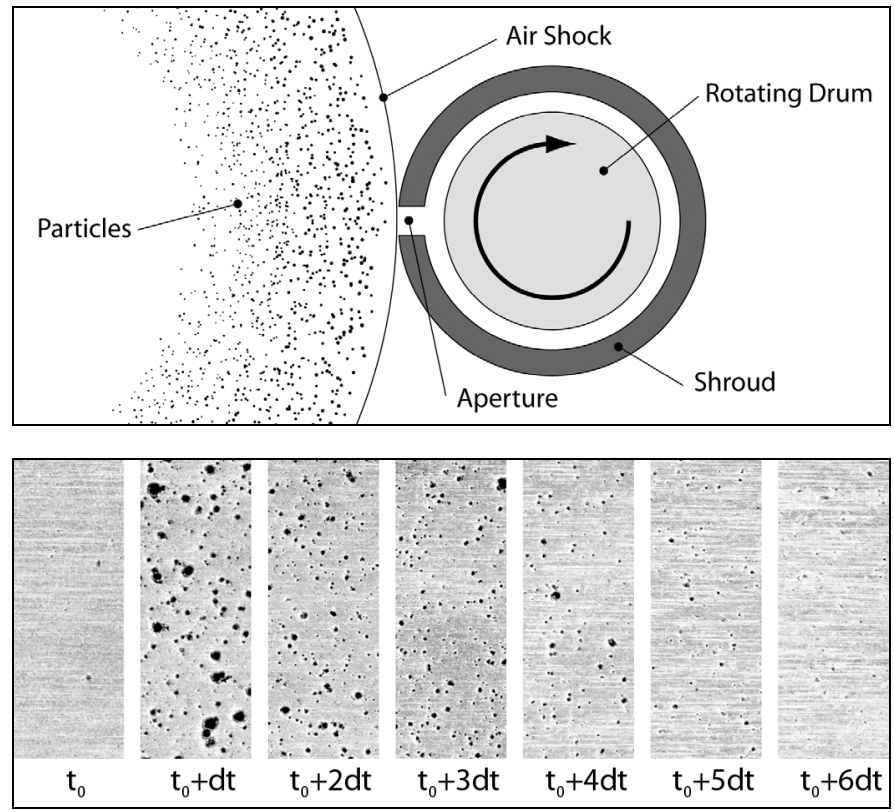

Figure 2: The Particle Streak Recorder (above) and typical impact surface images (below).

The data produced by the particle streak recorder include a collection of impact surface images that need to be analyzed in order to produce a history of number density and area density of holes. This analysis technique can be conducted in a number of ways. For large, uniformly sized particulate (e.g. uniform mm-scale spheres), it is possible to visually inspect the surface at regular spatial intervals (which correspond to regular temporal intervals). This technique has been employed in, e.g., [5]. For smaller, non-uniform-size particulate (as in the bottom of Fig. 2), the number and sizes of impact locations preclude the use of a visual inspection technique and an image processing technique must be used to analyze the data.

This technique normally consists of three steps: the first is the production of a series of digital micrographs of the surface of the drum. These micrographs must be of appropriate magnification to capture both the smallest feature of interest (normally the smallest particle size) as well as the smallest time span of interest. This image must then be converted into a two-color "binary image" where one color represents undisturbed surface and the other represents an impact hole (or crater for lower-energy particulate). Finally, this binary image is then analyzed for connectivity among pixels to determine the statistics associated with the impact locations. These statistics include parameters such as number of impact points, mean impact point size, and $\max / \mathrm{min}$ impact point size. These statistics, when collected at various spatial locations across the drum surface, provide the time-history of the number and size of particulate that passed through the aperture. 


\section{Analysis technique}

Analyzing the data collected using the momentum trap technique is straightforward: the throw distance is directly proportional to the total impulse delivered to the block. The mass of the block and geometry of the setup define the constant of proportionality. The analysis technique employed for the particle streak recorder is somewhat more complicated, however, due to the uncertainties associated with the measurement technique. Though the particle number density is known as a function of time, the particle speeds are not. As such, the time history of the particle speeds must be inferred from the particle streak recorder data and a suitable model for their behavior. The methodology described here begins with an appropriate form of the momentum flux rate equation for the solid-phase particles and postulates models for the terms therein.

\subsection{Particle mass and momentum flux}

Before continuing with the form of the momentum equation used for the solidphase particles it is instructive to review the momentum flux rate equations for continuum (i.e. single-phase) flows. For this class of flows, the total mass flux rate per unit area is given by

$$
\frac{d m_{A, c}}{d t}=\rho_{c} V_{c}
$$

where the subscripts $c$ are used here to denote an equivalent-continuum description of the solid-phase particulate flow. Note that the mass $m_{A, c}$ is the mass flux per unit area in the equivalent continuum description. While this description is useful for a continuum problem, it is more instructive to write the mass flux in terms of the number density of particles in a particulate blast flow. In this case, the mass flux is characterized by a number density of particles per unit area, $N_{A}$, and an average particle mass, $m_{p}$. Using this description the mass flux rate is given by

$$
\frac{d m_{A}}{d t}=m_{p} \frac{d N_{A}}{d t}
$$

where $m_{A}$ is the mass flux of particles per unit area. The momentum flux rate associated with this mass flux rate is simply the product of the mass flux rate and speed of the moving mass. For the equivalent continuum case, it is the wellknown parameter $\rho_{c} V_{c}^{2}$. For the solid-phase particulate the expression is given as

$$
\frac{d p_{A}}{d t}=m_{p} V_{p} \frac{d N_{A}}{d t}
$$


where $p_{A}$ is the momentum flux per unit area and $V_{p}$ is a representative average speed of the particulate. Note that $V_{p}$ is, in general, a function of time.

\subsection{Models for particle number density and speed}

Eq. (5) provides the relationship between momentum flux rate, particle number density flux rate, and particle speed. In order to conduct the analysis that follows these parameters must be expressed in analytical form. Insight gained from previous testing has shown that these parameters behave roughly as the structure of the air blast wave and, for this reason, it is reasonable to approximate their behavior using decaying exponentials (as for air blast properties in, e.g., [6]). For the number density flux rate this relationship can be expressed as

$$
\frac{d N_{A}}{d t}=A_{N} t^{2} e^{-k_{1}\left(t-t_{0}\right)} \text { for } t \geq t_{0}
$$

where $A_{N}, k_{l}$, and $t_{0}$ are chosen to provide an appropriate representation of the measured particle number density flux rate. The $t^{2}$ term is included to allow for the observed behavior of an initial rise in the particle number density flux rate; this rise is quickly overcome, however, by the decaying exponential behavior. Note that $t_{0}$ is the time at which the particulate first arrives at the measurement location. Note also that the time $t$ is taken as time from detonation and that the expression in Eq. (6) is only valid for times greater than or equal to the arrival time $t_{0}$.

The particle speed can be modeled using a similar approach but with a different modification to account for behavior in the early time. Because the solid-phase particulate is generally a high-density material, it is likely that it suffers relatively little momentum change after the detonation process provides its initial speed. As such, the particulate is likely to remain at a constant speed as it moves outward after the detonation. This behavior will begin to change, however, as the particulate moves out of the high induced velocities behind the air shock wave. In this case, the particles will move either ahead of the shock or fall far behind and the drag on the particulate is likely to be substantial and cause a significant reduction in particle speed. The location at which this behavior is observed will be different for different multiphase blast fields; it is a necessary consideration in applying the model given here. In practice, the model described below will be valid (to first approximation) so long as the particulate remains behind the initial shock. The model captures the decay due to particles falling behind the shock but assumes that the initial portion of the particulate blast is contained in the air shock structure.

If the constant-initial-speed approximation is appropriate then the initial particulate speed at any standoff $d$ is given simply by $d / t_{0}$ where $t_{0}$ is the time after detonation at which the particulate reaches distance $d$. Allowing for decay from this initial value then gives a particle speed function of the form 


$$
V_{p}=\frac{d}{t} e^{-k_{2}\left(t-t_{0}\right)} \text { for } t \geq t_{0}
$$

where $k_{2}$ is chosen to provide a good match to the observed data. This model simply states that the initial particle speed at distance $d$ is equal to the initial particle speed after detonation followed by a decay given by the combination of the decaying exponential and $1 / t$ behavior.

\subsection{Determining the values of $A_{N}, k_{1}$ and $k_{2}$}

The methodology described here requires an estimate for three parameters: $A_{N}$, $k_{1}$, and $k_{2}$ in addition to a measure of the particulate arrival time $t_{0}$. All of these parameters are available from the data collected by the particle streak recorder. The value of $t_{0}$ can be measured using a variety of techniques but is usually achieved by including some type of timing fiducial on the rotating drum. This fiducial is measured relative to the detonation time (using, e.g., a once-perrevolution signal recorded on a data acquisition system with timing referenced to the detonation time) and is marked on the drum. During the analysis procedure, the data are collected using this fiducial mark as a timing reference, thereby allowing a determination of the time at which the particulate first arrives at the particle streak recorder. The values of $A_{n}$ and $k_{1}$ are determined by fitting the measured data to the form given in Eq. (6).

The value of $k_{2}$ is more difficult to determine but can be approximated if the "minimum marking speed" $V_{\min }$ of the particulate is known. The minimum marking speed is the lowest speed that will produce evidence of an impact on the rotating drum. This speed is a function of several parameters including particle mass, particle density, particle shape, and drum material. There are a number of impact theories and/or computational methods available to determine the value of $V_{\min }$ given the combination of particle and drum properties. It is important to note that the methodology described here captures only the properties of the particulate with speed greater than $V_{\min }$ (because slower particulate does not produce measurable data). As such, the combination of particle properties and rotating drum properties must be chosen carefully to make certain that the range of measured speeds (i.e. $V_{\min }$ and greater) captures the preponderance of the momentum and energy associated with the solid-phase particulate. If $V_{\min }$ is too high, a significant portion of the momentum and energy flux will not be measured and the methodology will produce erroneous results.

The particle streak recorder data show both the start time $t_{0}$ and end time $t_{l}$ of the particle impacts. As described above the end time $t_{l}$ corresponds to the minimum marking speed and can be used in the particle speed equation, Eq. (7), to give

$$
V_{\min }=\frac{d}{t_{1}} e^{-k_{2}\left(t_{1}-t_{0}\right)}
$$

or 


$$
k_{2}=\frac{1}{t_{0}-t_{1}} \ln \left(\frac{V_{\min } t_{1}}{d}\right)
$$

Eq. (9), then, gives the value of $k_{2}$ for given values of $V_{\min }$ and $t_{1}$.

\subsection{Computing the impulse per unit area}

The impulse per unit area produced by the solid particulate will be the integral of the momentum flux rate equation given in Eq. (5). In other words,

$$
I_{A}=\int_{t_{0}}^{\infty} \frac{d p_{A}}{d t} d t
$$

Using the form of the number density flux relationship given in Eq. (6) and particle speed given in Eq. (7) gives the impulse per unit area as

$$
I_{A}=A_{N} m_{p} d e^{\left(k_{1}+k_{2}\right) t_{0}} \int_{t_{0}}^{\infty} t e^{-\left(k_{1}+k_{2}\right) t} d t
$$

which can be integrated to give

$$
I_{A}=\frac{m_{p} d A_{N}\left[1+\left(k_{1}+k_{2}\right) t_{0}\right]}{\left(k_{1}+k_{2}\right)^{2}}
$$

Once the values of $A_{N}, k_{1}$, and $k_{2}$ are determined from the data, Eq. (12) can be used to compute the impulse per unit area associated with the particle impacts.

\section{Discussion}

The accuracy of Eq. (12) is limited by the assumptions about the behavior of the particles. This accuracy can be improved by requiring the analysis to match the data collected by the momentum trap technique. However, the momentum trap technique captures the impulse produced by both the solid-phase and gas-phase loads; as such, the value of $I_{A}$ as computed in Eq. (12) should be less than the impulse per unit area measured using the momentum trap technique. In order to provide a rigorous check against measured data, the momentum trap technique must be able to distinguish between solid-phase and gas-phase loads.

This requirement can be addressed in a number of ways, the simplest of which is to provide a gas-phase pressure measurement on the momentum trap. This is most often achieved using a pressure gauge located behind a conical 
particle stripper. The stripper serves to remove the particles from the flow in the region of the pressure gauge, thereby preventing damage to the gauge and allowing a gas-phase-only measurement. However, the presence of the conical stripper is a disturbance to the flow and must be accounted for. Shock tube testing has shown that a conical stripper will produce significant changes in peak pressure (as much as $50 \%$ for some configurations) but that the impulse values are relatively unaffected ( $10 \%$ or less).

Beyond this correction is the correction required for the fact that the gasphase pressures produce a load in the direction opposite the blast wave direction once the shock passes the momentum trap. The air shock loads on the rear of the momentum trap serve to reduce the overall impulse delivered to the block (i.e. they load the block in the opposite direction of the initial blast). A single pressure gauge on the front of the momentum trap will produce a measurement that is much higher than the actual gas-phase impulse as a result of the back-face loading. It is usually possible, however, to create a correction for this phenomenon using an experimental or numerical approach.

The momentum trap technique, alone, can provide separate measurements of solid-phase and gas-phase impulse. However, it provides no temporal resolution and is limited by assumptions about the behavior of the air shock as it interacts with the block and particle stripper. Likewise, the particle streak recorder technique is limited by assumptions about the behavior of the particulate but it is able to provide a time-history of the loads produced by the solid-phase particulate. It cannot, however, provide gas-phase loads. The combination of the techniques, in conjunction with the methodology described here, provides a higher-confidence measurement than can be produced by either technique, alone.

\section{Conclusions}

This paper has presented a methodology that allows for measurements of momentum flux in two-phase blast flows. It outlined both the experimental apparatus and analysis techniques that are required to conduct such measurements. The paper has also discussed the validity of the approaches outlined here and has provided guidance on the circumstances under which the methodology is appropriate.

\section{Acknowledgements}

This work was jointly sponsored by the United States Office of Naval Research and the United States Office of the Undersecretary of Defense/Land Warfare and Munitions. In particular, Dr. Judah Goldwasser provided excellent support and guidance for the work presented here. The experimental apparatus was designed in conjunction with a number of contributors, including Sean Treadway, Mitch Moffett, Greg Larsen, and Perry Fridley. 


\section{References}

[1] Ames, R.G.; Murphy, M.J.; Groves, S.E.; Cunard, D.; "Diagnostics for Multiple-Phase Blast Flows", in Proceedings of the $3^{\text {rd }}$ International Conference on Fluid-Structure Interaction, September, 2005, La Coruna, Spain

[2] Hopkinson, B.; Proc. Roy. Soc. A, v. 87, 498; 1905

[3] Held, M; "Impulse Method for the Blast Contour of Cylindrical High Explosive Charges"; Propellants, Explosives, Pyrotechnics, Vol. 24, 1999

[4] Held, M; "Improved Momentum Method"; Propellants, Explosives, Pyrotechnics, Vol. 26, 2001

[5] Frost, D; Zhang, F et al.; "Near-Field Impulse Effects from Detonation of Heterogeneous Explosives"; in Proceedings of the Conference on Shock Compression of Condensed Matter, 2002

[6] Kinney, G.F.; Graham, K.J.; Explosive Shocks in Air; $2^{\text {nd }}$ Ed., SpringerVerlag, New York (1985) 